\title{
Prevalence of colonization by Streptococcus agalactiae among pregnant women in Bukavu, Democratic Republic of the Congo
}

\author{
Kashosi T Mitima ${ }^{1}$, Steve Ntamako ${ }^{2}$, Achippe M Birindwa ${ }^{3}$, Ntakwinja Mukanire ${ }^{4}$, John M Kivukuto ${ }^{5}$, \\ Kibendelwa Tsongo ${ }^{2}$, Kanigula Mubagwa ${ }^{6,7}$ \\ ${ }^{1}$ Biomedical Research and Public Health Laboratory, Université Evangélique en Afrique, Bukavu, DR Congo \\ ${ }^{2}$ Department of Internal Medicine and Infectious Diseases, Panzi Hospital, Bukavu, DR Congo \\ ${ }^{3}$ Department of Pediatrics, Panzi Hospital, Bukavu, DR Congo \\ ${ }^{4}$ Department of Gynecology and Obstetrics, Panzi Hospital, Bukavu, DR Congo \\ ${ }^{5}$ Médecins d'Afrique, Coordination-Europe, Savigny Sur Orge, France \\ ${ }^{6}$ International Center for Advanced Research and Training (ICART), Bukavu, DR Congo \\ ${ }^{7}$ Department of Cardiovascular Sciences, University of Leuven, Leuven, Belgium
}

\begin{abstract}
Introduction: Maternal vaginal colonization by Streptococcus agalactiae (GBS) has an important impact on neonatal health but has not been studied in the Democratic Republic of Congo. The aim of this study was to determine its frequency and influencing factors.

Methodology: Vaginal samples $(n=509)$ for bacteriological analysis were collected from women in Bukavu, eastern DR Congo, during their third trimester of pregnancy, along with information about age, education and socio-economic status, and medical and obstetricgynecological history.

Results: The overall GBS colonization rate was $20 \%$. Colonization was significantly associated with low education, history of urinary infection during the pregnancy, history of premature childbirth or abortion, and HIV-positive serology, but was not significantly associated with socio-economic level or parity.

Conclusions: The GBS colonization rate is similar to that found elsewhere on the continent. Further studies, with follow-up of neonates of infected mothers and evaluation of prevention/treatment strategies, are needed.
\end{abstract}

Key words: Streptococcus B; vaginal; colonization; pregnancy.

J Infect Dev Ctries 2014; 8(9):1195-1200. doi:10.3855/jidc.5030

(Received 24 March 2014 - Accepted 23 June 2014)

Copyright $(2014$ Mitima et al. This is an open-access article distributed under the Creative Commons Attribution License, which permits unrestricted use, distribution, and reproduction in any medium, provided the original work is properly cited.

\section{Introduction}

Streptococcus agalactiae is a Gram-positive bacterium belonging to Lancefield's group B streptococci (GBS). It is responsible for invasive infections mainly in pregnant women and infants $[1,2]$; it has been implicated in maternal infections such as endometritis, chorio-amniotitis, and puerperal infections of the uro-genital tract, as well as in neonatal pneumonia, meningitis, and septicemia [3]. Given this pathogenic role and the relatively frequent vaginal colonization with GBS, screening strategies have been proposed in several countries around the world, with the view to initiate prevention during the third trimester of pregnancy [3] or intrapartum treatment to avoid mother-to-newborn transmission [4]. In most countries in Africa, and in the Democratic Republic of Congo (DRC) in particular, due to the lack of a national policy for systematic screening of GBS carriage, no data on prevalence, mortality, and risk factors of this colonization have been reported to date. Yet, the neonatal mortality in these countries remains high. Indeed, the DRC is among the 12 countries in the world where neonatal mortality is greater than 39 per 1,000 living births [5]. In the specific context of developing countries, where hygiene and diagnostic conditions can be poor, it is not known to what extent a mother's colonization by GBS might be linked to adverse pregnancy outcomes or to the high rates of neonatal diseases. It is with this perspective that we conducted this study, in order to provide preliminary data for the public health issue of possible infectious causes of the high neonatal mortality. The aim of this study was, therefore, to assess the prevalence of GBS vaginal colonization among pregnant women attending prenatal care in selected healthcare centers within the 
city of Bukavu, Province of South-Kivu, in the eastern part of the DR Congo.

\section{Methodology}

The study was carried out in accordance with the ethical standards set out by the Declaration of Helsinki and in agreement with local institutional ethical guidelines.

This was a prospective cross-sectional study. It was conducted for one year, starting from 1 June 2012 to 31 May 2013, in 12 healthcare centers of Bukavu (4 in the health zone of Kadutu: Cimpunda, Uzima, CBCA Nyamugo, and Maria; 4 in the health zone of Bagira: Bagira, Nyamuhinga, Burhiba, and Mushekere; and 4 in the health zone of Ibanda: Panzi Hospital, Malkia wa Amani, 8th CEPAC Chahi, and Cidasa). The total population covered by these health centers was 272,804 , representing $27.6 \%$ of the whole population (987,592 inhabitants) of this urban environment. Sampling was exhaustive; vaginal samples were collected from all eligible pregnant women attending prenatal care in the last trimester of their pregnancies who gave informed consent. Women with genital fistulae and those taking antibiotics were excluded from the study. The following information was also collected through direct interview of the women: age, education, and socio-economic level (based on household income), medical and gynecological-obstetric history, including parity, urogenital tract infections during the current pregnancy, and results of previous HIV screening. Bacteriological sample collection was done using a sterile cotton swab inserted into the vaginal cavity through a speculum's opening. Swabs were immersed in sterile transport media (Portagerm, BioMérieux, Marcy l'Etoile, France) and were sent to the laboratory in a box insulated with ice packs within a maximum of three hours. In the laboratory, each swab was inoculated immediately on a culture medium made of blood agar containing nalidixic acid $(15 \mathrm{mg} / \mathrm{mL})$ and colistin $(10$ $\mu \mathrm{g} / \mathrm{mL}$ ). The culture dishes were incubated at $37^{\circ} \mathrm{C}$ under $\mathrm{CO}_{2}$ for 48 hours, with readings done every 24 hours. When beta-hemolytic colonies appeared within 24-48 hours of incubation under $\mathrm{CO}_{2}$, Gram staining was performed, followed by a catalase test, a hippurate test, and a Christie-Atkins-Munch-Petersen (CAMP) test using a Staphylococcus aureus strain. The catalase test was performed as follows: a drop of $10 \%$ hydrogen peroxide $\left(\mathrm{H}_{2} \mathrm{O}_{2}\right)$ was placed on a microscope glass slide; a sample of the beta-hemolytic colony was collected using a cooled sterile loop, and was added to and mixed with the $\mathrm{H}_{2} \mathrm{O}_{2}$ drop. The formation (positive reaction) or absence (negative reaction) of air bubbles was monitored. The hippurate test, aimed at detecting the presence of hippurate hydrolases, was performed using a rapid test kit (Mast Diagnostic, Amiens, France) according to the supplier's instructions as follows: an organism suspension with density corresponding to the McFarland standard no. 5 was prepared in Mast Peptone Water DM185 liquid growth medium; $0.5 \mathrm{~mL}$ of the bacterial suspension was added to a sterile, otherwise fully sealed, hippurate-containing tube (supplied as part of the kit), shaken gently and incubated at $37^{\circ} \mathrm{C}$ for 4 hours; two drops of Ninhydrin development reagent (also part of the kit) were added without shaking. The mixture was kept at room temperature for 15 minutes. The appearance (positive reaction) or absence (negative reaction) of a violet color was monitored. The CAMP test, which is based on the fact that group B hemolytic Streptococcus displays satellitism with $S$. aureus, was performed as follows: $S$. aureus was inoculated in a blood-based culture medium in a straight line or streak; GBS suspect colony was inoculated in the same medium using a streak perpendicular to the first one. Enhanced hemolysis by $S$. aureus was induced at the junction between the two streaks, forming a half-moon or arrowhead image. Only Gram-positive colonies giving a negative catalase reaction were further identified by the hippurate and CAMP tests. At the end, the Lancefield serological grouping was performed by an anti-B latex agglutination test (Slidex Strepto-Kit, BioMérieux). This test was performed as follows: a dense (2-3 colonies) bacterial suspension was made in $0.5 \mathrm{~mL}$ of a medium containing surface antigen extraction enzyme; the suspension was kept at $42^{\circ} \mathrm{C}$ for at least 15 minutes; a drop of a medium containing latex and one of the six (A, B, C, D, F, G) groups of antibodies was placed on a piece of cardboard with a black background; then a drop of the extraction suspension was added and mixed for about 1 minute. The appearance of agglutination in the drop identified the Streptococcus group corresponding to the antibody used.

Statistical analysis of the data was made using the EPI INFO software, version 3.4.1. Association of GBS infection with any risk factor was analyzed by calculating the odds ratio (OR), with a confidence interval of $95 \%(95 \% \mathrm{CI})$. Associations that gave an OR greater than 1 with a $\mathrm{P}$ value of less than 0.05 were considered significant. 


\section{Results}

During the study period, 731 visits for last trimester prenatal care took place in the 12 selected health centers. Of those women, 178 were excluded and 44 of the 553 eligible candidates did not consent to the study. Therefore, bacteriological samples were collected from 509 pregnant women who agreed to participate to the screening.

Table 1 presents the socio-demographic distribution of the patients, grouped according to health zone. As expected, the majority of the pregnant women were between 18 and 35 years of age. However, nearly one-quarter (24\%) were older than 35 years and $10 \%$ were younger than 18 years of age. Primiparae represented only $35 \%$ of the pregnant women. Two other social variables analyzed were education and socio-economic level. Most (64\%) of the women had reached the secondary school education level and could be considered to be literate. Nevertheless, $71 \%$ of the pregnant women had a very low socio-economic status and were without an identifiable income source.

Table 2 shows the average prevalence of vaginal colonization with GBS in the three health zones. The overall prevalence was $20 \%$, with no difference between the three city areas $(19 \%$ in Ibanda, $20 \%$ in Kadutu, and $21 \%$ in Bagira). History collected by a simple questionnaire indicated that only 18 women (4\%) had tested positive for HIV in this sample. Similarly, $12 \%$ of the women reported a history of previous spontaneous abortion and/or premature delivery. Finally, almost one-third (29\%) had had a history of urinary tract infections during the current pregnancy.

From univariate analysis of the results, as shown in Table 3, factors associated with a significant risk $(\mathrm{p}<$ $0.05)$ of vaginal colonization with GBS were, in ascending order: a low education level (OR: $2.5 ; 95 \%$ CI: $1.57-3.97)$, a history of urinary tract infection during the pregnancy (OR: 3.97; 95\% CI: 2.46-6.39), a

Table 1. Grouping of the pregnant women according to age, parity, education level, and socio-economic status

\begin{tabular}{|c|c|c|c|c|c|}
\hline Parameter & $\begin{array}{l}\text { Kadutu } \\
\mathrm{n}=\mathbf{2 7 3}\end{array}$ & $\begin{array}{l}\text { Bagira } \\
\mathrm{n}=121\end{array}$ & & $\begin{array}{l}\text { Ibanda } \\
\mathrm{n}=115\end{array}$ & $\begin{array}{c}\text { Total } \\
\mathrm{N}=509(\%) \\
\end{array}$ \\
\hline$<18$ years & 34 & 11 & & 6 & $51(10)$ \\
\hline$>35$ years & 57 & 32 & & 32 & $121(24)$ \\
\hline \multicolumn{6}{|l|}{ Parity } \\
\hline Primiparae & 93 & 45 & & 38 & $176(35)$ \\
\hline \multicolumn{6}{|c|}{ Education level } \\
\hline$\leq$ Primary & 93 & 59 & & 33 & $185(36)$ \\
\hline$\geq$ Secondary & 180 & 62 & & 82 & $324(64)$ \\
\hline \multicolumn{6}{|c|}{ Socio-economic level } \\
\hline Low & 188 & 85 & & 89 & $362(71)$ \\
\hline Middle & 85 & 36 & & 26 & $147(29)$ \\
\hline \multicolumn{2}{|c|}{ Parameter } & $\begin{array}{l}\text { Kadutu } \\
\mathrm{n}=\mathbf{2 7 3}\end{array}$ & $\begin{array}{l}\text { Bagira } \\
\mathrm{n}=121\end{array}$ & $\begin{array}{l}\text { Ibanda } \\
\mathrm{n}=115\end{array}$ & $\begin{array}{c}\text { Total } \\
\mathrm{N}=\mathbf{5 0 9}(\%) \\
\end{array}$ \\
\hline \multicolumn{6}{|c|}{ Urinary tract infections during the pregnancy } \\
\hline Yes & & 92 & 25 & 29 & $146(29)$ \\
\hline No & & 181 & 96 & 86 & $363(71)$ \\
\hline \multicolumn{6}{|c|}{ Abortion and/or premature delivery } \\
\hline Yes & & 36 & 15 & 12 & $63(12)$ \\
\hline No & & 237 & 106 & 103 & $446(88)$ \\
\hline \multicolumn{6}{|c|}{ HIV serological status } \\
\hline Positive & & 10 & 4 & 4 & $18(4)$ \\
\hline Negative & & 263 & 117 & 111 & $491(96)$ \\
\hline
\end{tabular}


positive HIV test (OR: 4.22; 95\% CI: 1.49-11.97), and a history of spontaneous abortion or premature delivery (OR: 8.19; 95\% CI: 4.49-15.01). In contrast, the young age of the pregnant women and their parity or socio-economic status were not found to be correlated $(\mathrm{OR}<1 ; \mathrm{p}>0.05)$ with the prevalence of vaginal colonization with GBS during the third trimester of pregnancy.

\section{Discussion}

The impact of female genital colonization with GBS during pregnancy on maternal and neonatal health is now well recognized and has been the focus on extensive research work in developed countries. However, considerably fewer studies have been conducted in African countries. The present study is the first one investigating GBS in a population of the DRC.

The majority of pregnant women included in this study were between 18 and 35 years of age. This age range corresponds to the reproductive period of women in most of developing countries. A significant percentage $(10 \%)$ of the participants were under 18 years of age, below the age when marriage is usually legally authorized, probably as a result of uncontrolled rural-to-urban exodus and increased urban promiscuity in a post-conflict context (marked by poverty and increased sexual violence) such as the one prevailing in the area of this study. About two-thirds of our respondents were multiparae, and there was a similar proportion of women with an education level equal to or greater than secondary level. This indicates that the majority of the women were likely to be well informed about pregnancy and hygiene. More than $70 \%$ of our respondents were of a low socio-economic status (with a family income of less than USD 100 a month for a general average of six individuals per family). This does not necessarily imply that the patients belonged mostly to the poorest section of the local population, as poverty and unemployment are general hallmarks of the population in the DRC.

Our findings reveal an average rate of colonization of $20 \%$ in pregnant women in the city of Bukavu. The rate appears uniform in all the three health zones of the city. Different colonization rates have been observed in various studies throughout the world, with prevalence of asymptomatic GBS carriage ranging over one order of magnitude, from $<3 \%$ to $>30 \%$ $[2,6,7]$. The colonization rate does not seem to be related to the socio-economic development of the country. An American study reported a vaginal carriage rate of GBS of $15.6 \%$ among women at 23-26 weeks gestational age [8]. On the European continent, the GBS carriage rate by pregnant women has also

Table 3. Association between various factors and GBS colonization

\begin{tabular}{|c|c|c|c|c|c|}
\hline Studied variables & $\begin{array}{c}\text { GBS carriage } \\
n=103\end{array}$ & $\begin{array}{c}\text { No GBS carriage } \\
n=406\end{array}$ & OR & $95 \% \mathrm{CI}$ & $\mathbf{P}$ \\
\hline \multicolumn{6}{|l|}{ Age } \\
\hline 18 years -35 years & 63 & 274 & 0.76 & $0.47-1.22$ & 0.22 \\
\hline \multicolumn{6}{|l|}{ Parity } \\
\hline Multiparae & 64 & 269 & 0.84 & $0.52-1.34$ & 0.43 \\
\hline Primiparae & 39 & 137 & & & \\
\hline$\leq$ Primary & 53 & 121 & 2.50 & $1.57-3.97$ & 0.00003 \\
\hline$\geq$ Secondary & 50 & 285 & & & \\
\hline \multicolumn{6}{|l|}{ Socio-economic level } \\
\hline Low & 58 & 304 & 0.43 & $0.27-0.69$ & 0.0002 \\
\hline Middle & 45 & 102 & & & \\
\hline \multicolumn{6}{|c|}{ Urinary tract infections during the pregnancy } \\
\hline Yes & 37 & 26 & 8.19 & $4.49-15.01$ & 0.0000000 \\
\hline No & 66 & 380 & & & \\
\hline \multicolumn{6}{|l|}{ HIV positive } \\
\hline Yes & 9 & 9 & 4.22 & $1.49-11.97$ & 0.001 \\
\hline No & 94 & 397 & & & \\
\hline
\end{tabular}

Results of univariate analysis. Odds ratios (OR), with confidence intervals of $95 \%$ ( $95 \% \mathrm{CI})$, were calculated. 
varied between different studies, even between countries sharing geographical proximity and similar socio-economic conditions (e.g., rates of $10 \%$ in France vs. $23.7 \%$ in Belgium) [9]. In developing countries, the prevalence of genital and anal carriage of GBS has been also reported to vary between $1 \%$ and $>30 \%$ [6]; further examples can be found in the works of Mavenyengwa et al. [10] and Sharmila et al. [11]. Given these differences, standardized screening methods have been proposed because part of the reported variations may reflect differences in methodology rather than possible real differences in prevalence. However, in general, prevalence rates on the African continent seem to be similar to those of developed countries [12]. In Morocco, one study found asymptomatic carriage rate of $23.3 \%$ [3]. Similar or higher rates were found in Southern Africa [10,12-14]. Our study, which is the first on this topic to be conducted in the DRC, had similar results. In the city of Bukavu, urinary tract infections during pregnancy, miscarriage, and premature birth history were associated with a high odds ratio for vaginal colonization in pregnant women. In this respect, our findings confirm those of other studies in Tunisia, in which a statistically significant association between GBS carriage and genital tract infections during pregnancy was found [15]. In contrast, other studies from Tunisia and from Morroco did not find any association with the following risk factors: age, school education level, parity, history of abortion or spontaneous miscarriage, history of extra-uterine pregnancy, fatal death in utero, preterm pyelonephritis, gestational diabetes, and twin pregnancy $[3,15,16]$.

Our study highlights the association of GBS vaginal colonization with HIV seropositivity. Although the association with HIV reported here might have been underestimated because HIV-positive status was based on history of tests carried out previous to this study, the finding is in agreement with the results of studies in Burkina Faso [17] and Zimbabwe [10], which also found a higher prevalence of GBS in pregnant women infected with HIV. However, such an association was not found in all previous studies $[13,18]$. The reasons for these discrepancies remain unclear. As far as the present study is concerned, one limitation about the strength of the correlation found is the low number of HIVpositive patients (18 out of 509). The prevalence (4\%) of HIV among the pregant women is in agreement with the rate in the general local population $(3.5 \%$; Panzi Hospital unpublished data). Larger numbers of pregnant women will be needed to further test this correlation.

As stated earlier, the relatively high rate of vaginal colonization with GBS and its pathogenicity have justified the implementation of screening, prevention, and treatment strategies $[2,4,7,12]$. Clinical practice guidelines have advocated intrapartum antibiotherapy to prevent GBS infection of the neonate, but the expected prevention of late-onset neonatal infection does not seem to be supported by conclusive data [4]. Currently, in Bukavu and elsewhere in the DRC and many other African countries, maternal intrapartum antibiotic or alternative treatments such as chlorhexidine irrigation are not part of the local clinical guidelines. Because the issue about the usefulness of current anti-GBS preventive or therapeutic pharmacological measures remains unresolved, it is important to analyze the impact of GBS in the specific context of low-income countries [6] such as the DRC, where the high fertility rate (more than six births per woman) may facilitate further testing of prevention measures.

The present study involved only an urban population; it will be of interest, therefore, to investigate some representative rural areas. Although data such as those of the present study are only descriptive, they may nevertheless be useful, as they can constitute the basis for an eventual intervention study on preventive and therapeutic measures against neonatal infection. In the unique environment of the $\mathrm{DRC}$, the risk/benefit ratio of such measures has not been evaluated and might be different from those in more developed environments. Given that the DRC has a very high neonatal mortality rate, and that data elsewhere suggest that neonates with GBS from maternal transmission present with exceptionally high infection risks [19], the results of the present study could set the stage for a good intervention study to resolve the issue of ante- or intrapartum treatment $\mathrm{risk} / \mathrm{benefit}$ ratio, or to test the effect of vaccination.

\section{Conclusions}

Vaginal colonization with Streptococcus agalactiae (GBS) is frequent among pregnant women in the city of Bukavu. Risk factors associated with this condition are low level of education, genito-urinary tract infections during pregnancy, HIV infection, and history of abortion and/or of premature delivery. The negative impact of GBS colonization has not been fully clarified, especially in countries where hygiene might be poor. Additional studies are needed to follow up the fate of pregnancies and the status of neonates 
born to mothers colonized by GBS. Such studies are needed to allow policy decision on the usefulness of screening, prevention, and treatment strategies.

\section{Acknowledgements}

This work was supported by an Evangelical University in Africa grant and by a University of Michigan Global Challenge grant. The content of this article is solely the responsibility of the authors and does not necessarily represent the official views of the Evangelical University in Africa, the Panzi Foundation, or the Panzi Hospital. We thank all the Congolese women who participated in this work. We also thank Mr. Ali Bitenda and Professor J. Miller for assistance.

\section{References}

1. Goubau P, Van Gompel A (2000) Repères en Microbiologie. Louvain: Garant. 391 p.

2. Le Doare K, Heath PT (2013) An overview of global GBS epidemiology. Vaccine 31 Suppl 4: D7-12.

3. Mahmoud M, Yahyaoui G, Benseddik N, Saadi M, Chaara H, Melhouf M (2011) Dépistage de streptocoque du groupe B au cours du troisième trimestre de grossesse au CHU Hassan II de Fès. Revue Tunisienne d'Infectiologie 5: 12-15.

4. Ohlsson A, Shah VS (2013) Intrapartum antibiotics for known maternal Group B streptococcal colonization. Cochrane Database Syst Rev 1: Cd007467.

5. Oestergaard MZ, Inoue M, Yoshida S, Mahanani WR, Gore FM, Cousens S, Lawn JE, Mathers CD (2011) Neonatal mortality levels for 193 countries in 2009 with trends since 1990: a systematic analysis of progress, projections, and priorities. PLoS Med 8: e1001080.

6. Stoll BJ, Schuchat A (1998) Maternal carriage of group B streptococci in developing countries. Pediatr Infect Dis J 17: 499-503.

7. Verani JR, McGee L, Schrag SJ (2010) Prevention of perinatal group B streptococcal disease--revised guidelines from CDC, 2010. MMWR Recomm Rep 59: 1-36.

8. Regan JA, Klebanoff MA, Nugent RP (1991) The epidemiology of group B streptococcal colonization in pregnancy. Vaginal Infections and Prematurity Study Group. Obstet Gynecol 77: 604-610.

9. Lorquet S, Melin P, Minon JM, Carpentier M, Gerday C, Rigo J, Foidart JM (2005) Group B streptococcus in the antenatal clinic and in the delivery room: a matter of systematic attitude. J Gynecol Obstet Biol Reprod (Paris) 34: 115-127.

10. Mavenyengwa RT, Moyo SR, Nordbo SA (2010) Streptococcus agalactiae colonization and correlation with HIV-1 and HBV seroprevalence in pregnant women from Zimbabwe. Eur J Obstet Gynecol Reprod Biol 150: 34-38.
11. Sharmila V, Joseph NM, Arun Babu T, Chaturvedula L, Sistla S (2011) Genital tract group B streptococcal colonization in pregnant women: a South Indian perspective. J Infect Dev Ctries 5: 592-595. doi:10.3855/jidc.1551.

12. Capan M, Mombo-Ngoma G, Akerey-Diop D, Basra A, Wurbel H, Lendamba W, Auer-Hackenberg L, Mackanga R, Melser J, Belard S, Ramharter M (2012) Epidemiology and management of group B streptococcal colonization during pregnancy in Africa. Wien Klin Wochenschr 124 Suppl 3: 1416.

13. Gray KJ, Kafulafula G, Matemba M, Kamdolozi M, Membe G, French N (2011) Group B Streptococcus and HIV infection in pregnant women, Malawi, 2008-2010. Emerg Infect Dis 17: 1932-1935.

14. Moyo SR, Mudzori J, Tswana SA, Maeland JA (2000) Prevalence, capsular type distribution, anthropometric and obstetric factors of group B Streptococcus (Streptococcus agalactiae) colonization in pregnancy. Cent Afr J Med 46: 115-120.

15. Ferjani A, Ben Abdallah $\mathrm{H}$, Ben Saida N, Gozzi C, Boukadida J (2006) Vaginal colonization of the Streptococcus agalactiae in pregnant woman in Tunisia: risk factors and susceptibility of isolates to antibiotics. Bull Soc Pathol Exot 99: 99-102.

16. Jerbi M, Hidar S, Hannachi N, El Moueddeb S, Djebbari H, Boukadida J, Chaieb A, Khairi H (2007) [Risk factors for group B streptococcal colonization in pregnant women at term: prospective study of 294 cases]. Gynecol Obstet Fertil 35: 312-316.

17. Djigma F, Ouedraogo C, Sagna T, Ouermi D, Sanogo K, Bisseye C, Kabre A, Pietra V, Simpore J, Nikiema JB, Musumeci S (2011) HIV-infected women of Burkina Faso: a "reservoir" of mycoplasma infection. J Infect Dev Ctries 5: 176-181. doi:10.3855/jidc.950.

18. El Beitune P, Duarte G, Maffei CM, Quintana SM, De Sa Rosa ESAC, Nogueira AA (2006) Group B Streptococcus carriers among HIV-1 infected pregnant women: prevalence and risk factors. Eur J Obstet Gynecol Reprod Biol 128: 5458.

19. Yagupsky P, Menegus MA, Powell KR (1991) The changing spectrum of group B streptococcal disease in infants: an eleven-year experience in a tertiary care hospital. Pediatr Infect Dis J 10: 801-808.

\section{Corresponding author}

Professor Kanigula Mubagwa, MD PhD

International Center for Advanced Research and Training (ICART)

Ave. Mushununu, Panzi, Bukavu, DR Congo

Phone: +24 3975299968 and +32 16330808

Email: kanigula.mubagwa@med.kuleuven.be

Conflict of interests: No conflict of interests is declared. 